\author{
C. R. MILLER \\ Georgia College, Milledgeville, Ga. 31061 \\ and \\ G, R, SESSIONS \\ University of Georgia, Athens, Ga. 30601
}

different occasions prior to the present experiment. Females of the same age and strain served as mating partners. The females had been ovariectomized and were brought into estrus by an intramuscular injection of $0.1 \mathrm{mg}$ of estradiol benzoate $75 \mathrm{~h}$ prior to testing and an intramuscular injection of $1.0 \mathrm{mg}$ of progesterone $3 \mathrm{~h}$ prior to testing.

All animals were individually housed with constant access to food and water. The colony was maintained under an altered light-dark cycle, in which the lights came on at 12:00 midnight and went off at 12:00 noon. Testing was conducted in the early portion of the dark cycle, between 1:00 p.m. and 4:00 p.m.

\section{APPAK ATUS AND PROCEDURE}

The observation arena was a circular Plexiglas enclosure $45 \mathrm{~cm}$ in diam. The arena had a clear glass floor and was mounted in a frame so that a mirror could be positioned beneath the floor to afford the $E$ a ventral view of the animals. This allowed the $E$ to easily distinguish mounts (abortive copulatory attempts), intromissions, and ejaculations. An Esterline-Angus event recorder was used to record intromissions, ejaculations, and interresponse intervals.

Preceding each test, the male was allowed a 5 -min period to adapt to the apparatus. Each $S$ was tested on four different occasions with each test separated by a period of 7 days. In the first test, or pretest, each male was allowed to copulate without $E$ intervention until five ejaculations had been recorded. At that point, the test was terminated. For the second test, the males were divided randomly into two groups of seven animals each. One group was tested under the single delay condition and the other was tested under the multiple delay condition. In the single delay test, the female was removed, by hand, from the arena for a 60-sec period immediately after the male achieved the initial intromission. At the end of the 60-sec interval, the female was returned and the male was allowed to continue without interruption until two ejaculations were achieved, at which point the test was stopped. The multiple delay tests were procedurally identical to the single delay tests, except that the female was removed for $60 \mathrm{sec}$ after each intromission until the first ejaculation occurred. For the third test, the testing conditions for the two groups were reversed from those of the second test. Otherwise, there were no procedural differences between the second and the third tests. Finally, each male was given a postexperimental test under conditions identical to that used in the pretest. Since the previous study by 
Table 1

Mean Intromission Frequency Preceding the First Ejaculation ( F $\left._{1}\right)$, Mean Postejaculatory Interval (PEI), and Mean Intromission Frequency Preceding the Second Ejaculation $\left(\mathrm{IF}_{2}\right)$ in Each Test

\begin{tabular}{lrrr}
\hline & \multicolumn{3}{c}{ Measure } \\
\cline { 2 - 4 } \multicolumn{1}{c}{ Test } & \multicolumn{1}{c}{ IF $_{1}$} & PEI & $I_{2}$ \\
\hline Pretest & 16.5 & 62.4 & 3.7 \\
Single Delay & 7.7 & 36.3 & 3.5 \\
MIultiple Delay & 28.9 & 34.3 & 2.8 \\
Post test & 14.4 & 61.6 & 3.2 \\
\hline
\end{tabular}

Sessions \& Miller (1970) included controls for the effects of handling, such procedures were not included in the present study.

A record was made of the intromission frequency preceding the first ejaculation, the interval between the first ejaculation and the next i n tromission, termed the postejaculatory interval, and the number of intromissions required for the second ejaculation. Between-test comparisons of the frequency data were made by means of the Wilcoxon $T$ test (Siegel, 1956), and the interval data were analyzed by means of the $t$ ratio for matched observations.

\section{RESULTS}

During the multiple delay tests, 2 males achieved successive intromissions so quickly that it was not possible to remove the female before the next intromission occurred. The data from these two males were excluded from the study. For the remaining 12 males, there were no significant differences between the pre- and posttests on any measure employed. Consequently, the remaining between-tests comparisons used the pretest only as the basis for the comparison of the enforced delay effects.

When interintromission delays were imposed, there were statistically significant changes in the intromission frequency preceding the first ejaculation and in the postejaculatory interval. The intromission frequency of the second copulatory series was not significantly altered under any testing condition. When a 60 -sec delay was imposed after the first intromission only, the intromission frequency preceding the first ejaculation was significantly reduced in comparison to that observed during the pretest $(T=0, p<.01)$. In contrast, when a 60-sec delay was imposed after each intromission, the frequency of intromission preceding the first ejaculation was significantly increased $(\mathrm{T}=9, \mathrm{p}=.02)$. One male required 105 intromissions before ejaculation was achieved with multiple enforced delays. An inspection of the data from this male's last test to satiety, which took place 21 days prior to the beginning of the present study, showed that the animal had achieved 12 ejaculations prior to satiety, all of which had required only 34 intromissions.

The postejaculatory interval was significantly reduced from that recorded in the pretest in both the single delay condition $(\mathrm{t}=3.30, \mathrm{df}=$ $11, \mathrm{p}<.01$ ) and the multiple delay condition $(t=3.54, \mathrm{df}=11, \mathrm{p}<.01)$. The mean value for each measure in each testing condition is presented in Table 1.

Throughout the study, no male required longer than $15 \mathrm{sec}$ to renew copulatory activities when the female was returned at the end of the delay period. During the multiple delay tests, it was obvious that some males were maintaining intromission for an extended period of time. Some even began rhythmic pelvic thrusting without terminating vaginal penetration, a form of intromission also observed late in a mating test to satiety. Furthermore, when multiple delays were imposed, several males greatly abbreviated or eliminated altogether the autogenital grooming period typically observed between intromissions and, instead, attempted to remount the female immediately. To prevent the male from achieving two closely spaced intromissions, the $E$ had to remove the female very quickly.

\section{DISCUSSION}

The results of the present study confirm the findings of Sessions \& Miller (1970) that multiple 60-sec interintromission delays significantly increase the number of intromissions required by the male hamster to achieve ejaculation. However, a single delay of the same duration imposed only after the first intromission significantly reduced the number of intromissions required for ejaculation. This is in contrast to the results reported by Bermant (1964) on the effects of single and multiple enforced delays imposed on the male rat. In the Bermant study, multiple delays enhanced the frequency reduction effect produced by single delays imposed after the first or fourth intromission. In the present study, both delay conditions significantly reduced the postejaculatory interval, and a similar effect was observed in the male rat by Bermant.

As Bermant noted, a reduction in intromission frequency and a decrease in the postejaculatory interval suggest an increase in sexual efficiency. It also suggests a heightened state of sexual excitation in that less genital stimulation is required to produce ejaculation and the pacing of the male's responses is accelerated. For the hamster, the assumption of heightened sexual excitation is further supported by the immediacy of the male's response when the female is returned, the quickening of successive intromissions, and the change in the pattern and duration of the intromission response. Carlsson \& Larsson (1962) recorded significant increases in the duration of intromission following artificially prolonged interintromission intervals in the male rat and suggested that the increase in duration compensated in time for a decrease in intromission frequency.

Under the multiple delay conditions, the male hamster gave every indication of being in a high state of sexual excitement. This is particularly true in terms of the male's immediate response when the female was returned. Hard \& Larsson (1970) found that the mount attempt latency of the male rat following interintromission delay decreased as the enforced interval increased up to approximately $2 \mathrm{~min}$ and thereafter increased as the duration of the delay increased. This was interpreted as indicating that the excitatory effects of the preceding intromission begin to extinguish beyond a 2 -min delay, and, since intromission frequency did not show a corresponding increase as mount latency increased, it was concluded that the latency measure was a more sensitive index of changes in the excitatory effects of intromission. If this is true of the hamster as well, it may be concluded that the male hamster is in a highly excited state when multiple delays are imposed, since the latency of the male's response was quite brief. Nevertheless, significantly more intromissions were required for ejaculation than under ad lib copulatory conditions. While observing these males copulate, the impression given was that the animal was highly excited but lacked the necessary repetitive genital stimulation to achieve ejaculation. This suggests that the male's behavior is jointly determined by an interaction between a central mechanism and a peripheral mechanism. The primary source of stimulation for the peripheral mechanism seems to be genital stimulation, whereas the central mechanism is probably activated by several factors such as general arousal, stimuli from the receptive female, feedback from genital stimulation, as well as other factors. Whatever the nature of the effect of enforced delays, it appears to act on the central mechanism and can be offset by multiple interintromission delays, at least in the hamster. On the other hand, the central effects of a single delay appear to override the 
momentary postponement of genital stimulation resulting from a single delay.

The present study represents an initial investigation of the effect of delayed copulation on the mating pattern of the male hamster. More complete and parametric studies are needed for a better understanding of these effects.

\section{REFERENCES}

BEACH, F. A., \& WHALEN, R. E. Effects of intromission without ejaculation upon sexual behavior in male rats. Journal of
Comparative \& Physiological Psychology, $1959,52,476-481$

BERMANT, G. Effects of single and multiple enforced intercopulatory intervals on the sexual behavior of male rats. Journal of Comparative \& Physiological Psychology, 1964, 57. $398-403$.

BERMANT, G Copulation in rats. Psychology Today, 1967, 1,53-60.

CARLSSON, S. G., \& LARSSON, K Intromission frequency and intromission duration in the male rat mating behavior. Scandinavian Journal of Psychology, 1962. 3, 189-191.

GERALL A A Effect of interruption of copulation in male guinea pig sexual behavior. Psychological Reports, 1958, 4 , 215-221.

HARD, E. \& LARSSON, K. Effects of delaying intromissions on the male rat's mating behavior. Journal of Comparative \& Physiological Psychology, 1970, 70 , $413-416$.

LARSSON, $\mathrm{K}$. Conditioning and sexual behalior in the male albino rat Stockholm: Almquist \& Wiksell, 1956.

LARSSON, $K$. The effect of restraint upon copulatory behavior in the rat. Animal Behavior, 1959, 7, 23-25.

LARSSON, K. Excitatory effects of intromission in mating behavior of the male rat. Behavior, Leiden, 1960. 16 , 66-73.

SESSIONS, G. R., \& MILLER, C. R. The "enforced interval effect" in the male golden hamster. Paper presented at the Eastern Regional Conference on Reproductive Behavior, 1970, Coral Gables, Fla.

SIEGEL, S. Nonparametric statistics, New York: McGraw-Hill, 1956. 